\title{
Agôn
}

Revue des arts de la scène

Critiques | Saison 2015-2016

\section{La Procession, Compagnie Nacera Belaza}

Déployer un autre espace au MuCEM

\section{Frédérique Villemur}

\section{(2) OpenEdition}

Journals

Édition électronique

URL : http://journals.openedition.org/agon/3995

DOI : 10.4000/agon.3995

ISSN : 1961-8581

Éditeur

Association Agôn

Référence électronique

Frédérique Villemur, «La Procession, Compagnie Nacera Belaza », Agôn [En ligne], Critiques, mis en ligne le 10 février 2016, consulté le 23 septembre 2020. URL : http://journals.openedition.org/agon/ 3995 ; DOl : https://doi.org/10.4000/agon.3995

Ce document a été généré automatiquement le 23 septembre 2020

Association Agôn et les auteurs des articles 


\section{La Procession, Compagnie Nacera Belaza}

Déployer un autre espace au MuCEM

\section{Frédérique Villemur}

\section{RÉFÉRENCE}

Cie Nacera Belaza, La Procession, présenté au MuCEM à Marseille les 18 et 19 décembre 2015

MuCEM, Musée des Civilisations de l'Europe et de la Méditerranée

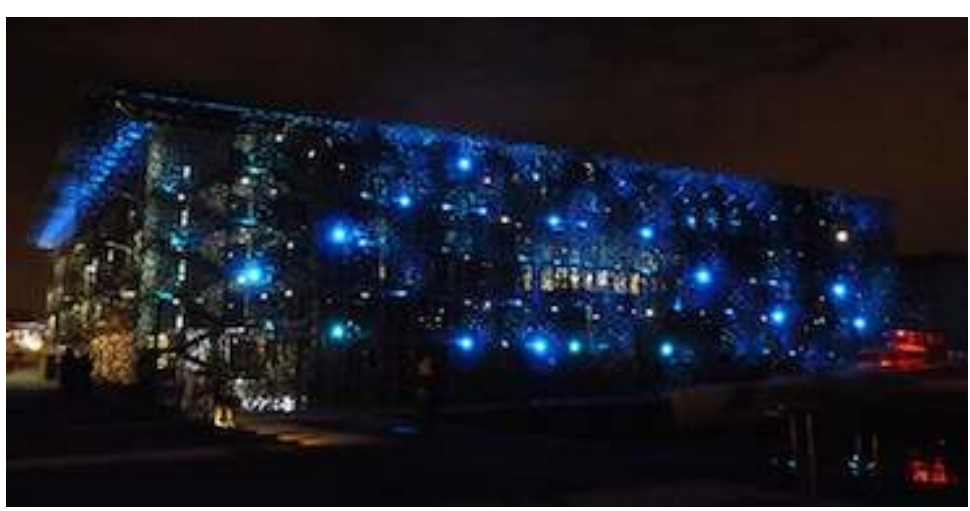

Frédérique Villemur

Cela se passe de nuit à Marseille, à l'entrée du Vieux Port, le J4 de Rudy Ricciotti, le MuCEM, Musée des Civilisations de l'Europe et de la Méditerranée scintille sous sa parure bleue, tout contre le Fort Saint-Jean dont la masse s'efface à cette heure. Des lucioles transpercent de-ci de-là sa mantille ajourée, irradiant d'une lumière secrète depuis le cœur du bâtiment la profondeur maritime à la proue du môle. On ne sait vers quel horizon flotte ce grand navire. Les points cardinaux se perdent comme se 
dispersent les limites de la ville. C'est à une descente du J4 en profondeur que nous invitent Nacera et Dalila Belaza. Elles ont choisi de ne pas chorégraphier comme on aurait pu s'y attendre les vides qui sertissent l'espace central de ces quelques $15000 \mathrm{~m} 2$ dont un tiers sont réservés aux expositions, les temporaires et la permanente, la «Galerie de la Méditerranée ». Le parcours de La Procession passe du «niveau 0 » au " niveau -1 ", de l'accueil vers le forum et l'auditorium, dans le creusement des douves. On est donc très loin de ce qu'on perçoit le jour, en tant que visiteur et spectateur du bâtiment que l'architecte définit ici lui-même de manière ethnocentrée et genrée :

«Une casbah verticale, un carré parfait de 72 mètres de côté, tenu par des structures arborescentes élancées, protégé par une enveloppe brise-soleil tel un moucharabieh. Minérale, tout en béton fibré, de couleur poussière mate, c'est une architecture de la maigreur, étirée comme les muscles tendus d'un coureur de fond, armée d'une délicatesse puissante et féminine. Elle renvoie à la métaphore de l'espace méditerranéen. [...] Comme dans une ziggourat, le visiteur se baladera, autour du musée, sur des rampes jusqu'au toit, entre l'air, le ciel, le soleil, les embruns, l'iode... Le paysage s'expose sous de multiples angles ${ }^{1}$.»

Car Nacera et Dalila Belaza donnent à découvrir l'envers du décor jouant moins sur les trames entre l'intérieur et l'extérieur que sur les zones de pénombre et de profondeur. C'est alors dans la dilatation du champ de perception que l'on prend la mesure, au sens musical du terme, de La Procession à laquelle elles nous invitent, une dilatation ouvrant sur un espace tout intérieur. Elles cheminent ainsi dans les dessous du bâtiment, en une descente occultant les jours des transparences de l'architecture, recomposant une cartographie des lieux vers les profondeurs marines, déployant un autre espace, mais ne s'éloignant guère de la densité du vide qui constitue le seul matériau véritable de l'architecte.

MuCEM, Musée des Civilisations de l'Europe et de la Méditerranée

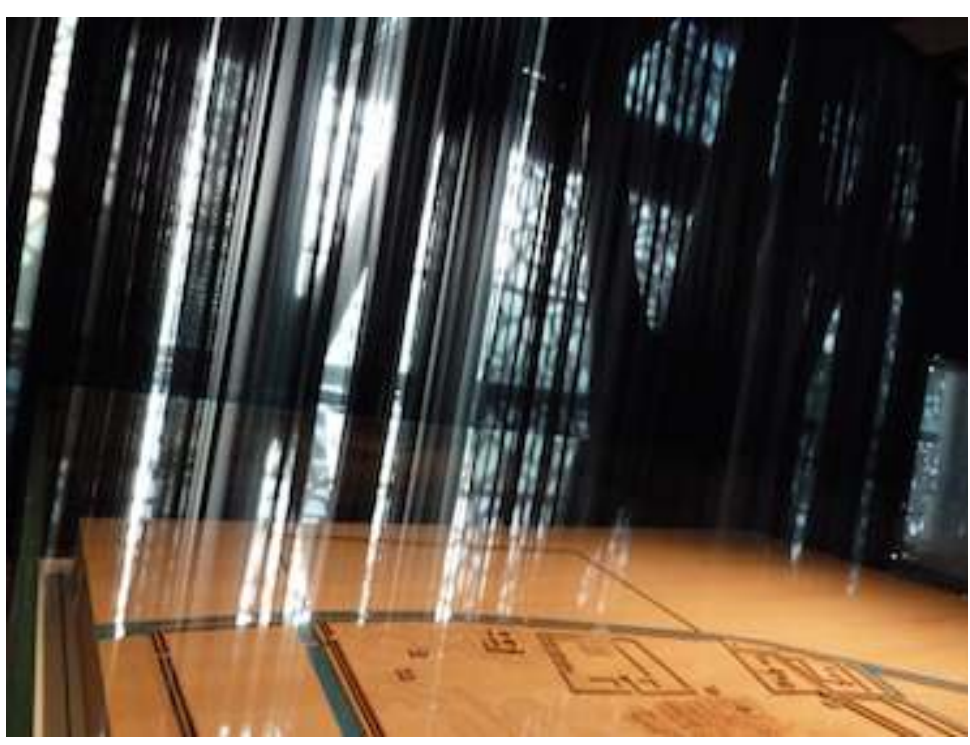

Frédérique Villemur

3 Dalila et Nacera Belaza ont répondu à l'invitation de Catherine Sentis et de Samar Kehdy à participer au cycle «Objets déplacés » du MuCEM ${ }^{2}$. On s'étonnera de voir leur danse associée à la notion d'objet, qui plus est d'objets au pluriel, tant apparaît éloigné de toute intention réifiante, de toute qualité mimétique leur geste dansé. «Ce projet 
était pour moi intéressant et assez inapproprié, avoue Nacera Belaza. Intéressant dans le fait d'insérer le public dans ce parcours, et inapproprié dans l'idée d'aller utiliser des objets, car antinomique par rapport au travail que je fais qui est dans l'abstraction. Je passe mon temps à vider, vider, vider des choses, la matière, l'espace ${ }^{3}$ ». S'agit-il d'une demande propre au contexte actuel, celui d'une recrudescence d'intérêt des musées envers la performance et la danse pour jeter un nouveau regard sur leurs collections, revisiter certains départements oubliés, saisir le corps en mouvement comme ultime tableau vivant, et donner corps aux objets comme à l'architecture ? Comme s'il y avait un désir de sortir de la communication sur l'architecture du J4 (sa contemporéanité médiatisée indissociable des opérations urbanistiques de la cité phocéenne), et d'en venir à ce qui constitue son fond, à la fois patrimoine matériel et immatériel des cultures méditerranéennes, présent de manière permanente au sein de la Galerie de la Méditerranée mais aussi dans les réserves du Centre de Conservation et de Ressources (CCR), construit en contrepoint du MuCEM par Corinne Vezzoni à la Belle de Mai. Avec la nécessité de puiser des ressources au CCR et de les redéployer au cœur du navire phocéen. Pour autant, s'agit-il dans ces déplacements de donner place aux objets ou de faire espace de gestes dansés? De donner sens au déplacement d'un geste muséal ou de donner lieu à chorégraphier une exposition tout autre ${ }^{4}$ ? La danse de Nacera Belaza privilégie le plateau nu, une chorégraphie sans objets sur scène, et plus encore une danse sans objet, refusant théâtralité et narrativité, dépouillant les gestes pour ne garder qu'une adresse lancée depuis une ouverture au vide. Il est vrai que la proposition de Samar Kehdy est arrivée à un moment où Nacera Belaza s'interrogeait sur la place du spectateur à donner à l'espace, et alors que Dalila Belaza dans son propre parcours questionnait le croisement des arts plastiques avec les arts vivants. De fait, la performance dansée avait pris le relais de la danse au plateau dans certaines expérimentations qu'elles avaient toutes deux déjà menées en 2012 au MaMa, le Musée d'art moderne d'Alger, parmi les sculptures et les peintures. C'était là pourtant dans un contexte tout à fait différent de commande publique : Nacera Belaza cherchait alors à replacer la danse dans la contemporanéité des arts en Algérie $^{5}$. Alors qu'au MuCEM, dans La Procession, incluant pour la première fois l'action du spectateur, le déplacement cherche à créer un autre espace.

«Objets déplacés » relève d'une critique des « objets» (autant de l'objet résultant de l'accumulation des objets dans la logique muséale que des usages sociaux associés à leurs dispositifs d'exposition) et vise donc à questionner l'exposition en tant que regroupement d'objets disparates dans un lieu donné. Ainsi, le geste dansé déborde-t-il l'exposition perçue comme unité fragmentaire d'espace. Il envahit l'espace du MuCEM en perturbant les vecteurs spatio-temporels des objets sélectionnés par la chorégraphe. Avec « Objets déplacés » il ne s'agit ni d'exposer des objets existants autrement ailleurs, ni de dévoiler les usages d'objets remisés en réserve, mais de donner sens à des objets trouvés en ce qui leur donne place, "fait espace», par le geste dansé. Il s'agit de percevoir hors de l'espace muséal dédié, et dans un temps éphémère, un objet d'une collection qui, déplacé, va se trouver reconfiguré, de même que l'espace qui l'environne. Chaque artiste (chorégraphe, metteur en scène) sollicité dans ce cycle ${ }^{6}$ doit présenter sa création en différents espaces du MuCEM, avec cette attente qu'un « objet du passé, déterré aujourd'hui, [puisse] éclairer d'un jour nouveau notre histoire présente $^{7} »$. Loin donc des modalités de la conservation et de l'exposition muséale, la proposition serait à chercher du côté de la "performance scénique ${ }^{8}$ ", voire même de l'« espace performatif ", nous verrons pourquoi. Et ce, dans un double déplacement, et 
de l'objet muséal, et du geste de l'art engageant une réflexion sur la création en arts vivants dans l'espace muséal.

Nacera et Dalila Belaza ont choisi pour La Procession trois objets dans les réserves du Centre de Conservation et de Ressources : un gros bloc de pierre circulaire troué, une épaisse structure de bois éventrée, un détail de mains ouvertes en prière sur une toile peinte 9 . Dans le parcours aucune indication ne renseigne leurs sources, leur datation ou leurs usages, seules leurs matières et les traces du passage du temps leur confère ce pouvoir d'être montrés de manière éphémère en un geste qui les rend très présents.

6 Tout se passe comme s'il s'agissait d'incorporer ce transfert d'objets déplacés, transport métaphorique ou non, de l'objet perçu et re-situé, donc interprété, par un geste à l'espace. Comme si on les découvrait pour la première fois en-dehors de la fixité muséale à laquelle ils sont attachés, qu'on les interprétait par un geste en mouvement dans l'espace leur faisant place, une autre place. Pro, "à la place de »: ils sont pleinement intégrés à $L a$ Procession, détournés de leurs usages et déplacés de leur lieu de conservation. Ainsi transférés du CCR vers le MuCEM et disposés hors des galeries d'exposition, ces objets se trouvent placés dans un parcours impliquant une progression dans l'espace partagé. Tout se passe comme s'ils pouvaient détenir par le choix opéré par la chorégraphe, le pouvoir de susciter ce lien que propose le geste dansé, qui est de relier les spectateurs entre eux. Il s'agit, dit Nacera Belaza, « d'inclure le spectateur dans le parcours, tout en prenant compte aussi bien des objets que de l'espace ». Le pari de cette rencontre était risqué, le geste performé pris à une certaine lenteur a atteint cet espace partagé. Avec La Procession les sœurs Belaza sont parvenues à révéler ce qui se joue dans une antériorité spatiale (pro), cet en-soi sur lequel elles travaillent, une intériorité, et ce qui se découvre dans une avancée - pro-cession marche au caractère rituel, cortège conçu comme l'émanation d'une trouvaille toute intérieure, mettant sur le devant (pro) la déposition d'un geste qui procède d'une antériorité vers un déplacement d'objet.

MuCEM, Musée des Civilisations de l'Europe et de la Méditerranée

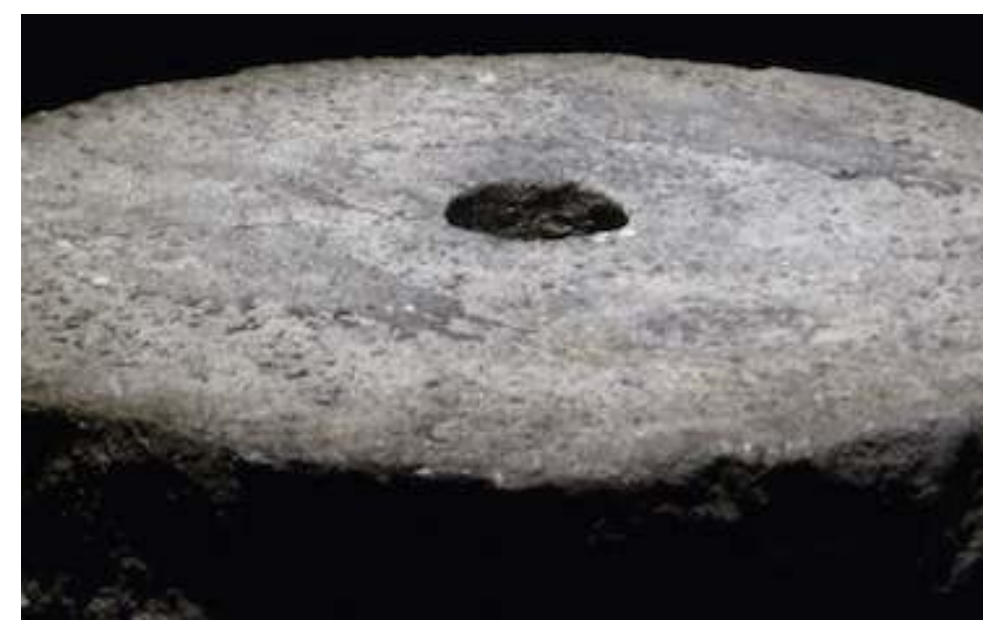

Frédérique Villemur

7 Nacera Belaza a disposé la pierre trouée au cœur du hall du MuCEM afin de prendre la mesure de la largeur de l'espace à partir de ce centre percé. Les spectateurs arrivant petit à petit du niveau -1 sont invités à épouser l'espace en se mettant en cercle autour d'elle, puis à prendre le sens commun d'une giration lente, pas à pas. Ils tournent 
autour de la trouée de cette matière dense qu'une douche de lumière perce. La pierre, le cercle, l'alentour: ce qui dans la journée n'est qu'un hall marqué d'ouvertures multiples et de croissement mobiles (visiteurs allant de la billetterie aux vestiaires vers les galeries d'exposition, de la restauration à la librairie) prend soudain dans une pénombre assumée où l'on voit à peine les images mouvantes de la vidéo d'un voile se soulevant par vagues entre les poteaux arborescents du hall, prend donc les qualités d'un espace non plus de services mais d'un espace qui rassemble. Les spectateurs se meuvent en immersion, comme pris à une gravité maritime. L'intériorisation du pas dans la marche et le mouvement de rotation troublent les repères spatiaux d'une architecture qui ne s'impose plus à eux, ils semblent flotter, et ne plus avoir à répondre d'un parcours fléché par une orientation informative. Seule la présence d'une matière érodée par de lointains usages aimante leur avancée.

MuCEM, Musée des Civilisations de l'Europe et de la Méditerranée

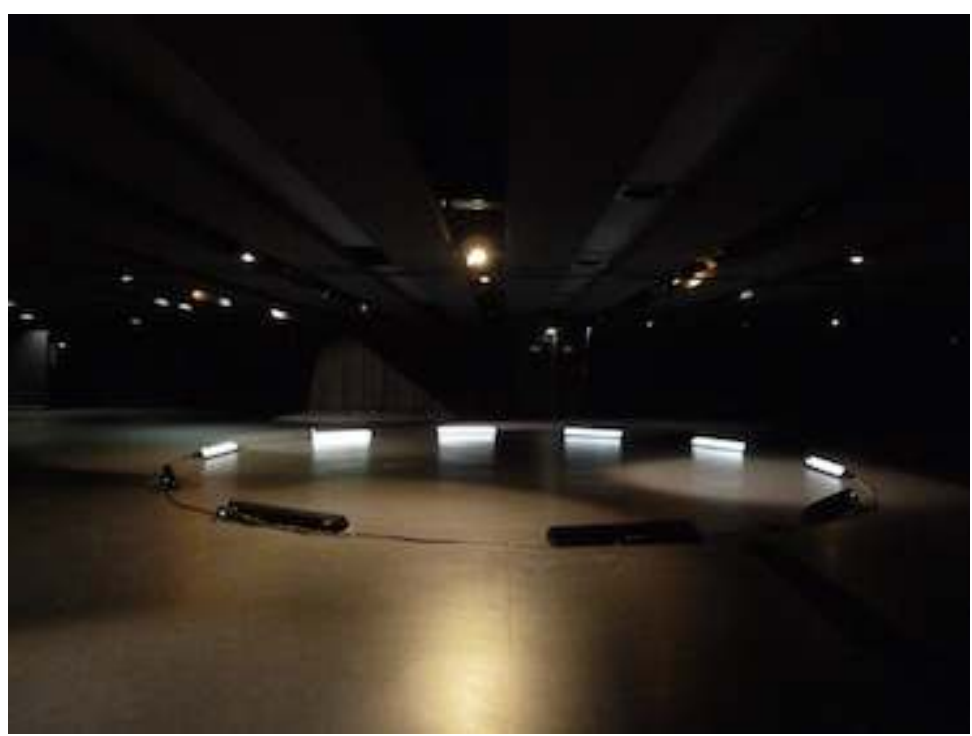

Frédérique Villemur

Guidés par des médiateurs culturels qui participent à La Procession, les spectateurs petit à petit entrés dans la marche processionnaire, descendent en sous-sol, au niveau du Forum, où un autre cercle les attend, scénique cette fois, délimité au sol par des néons de lumière blanche, dans lequel apparaît Nacera Belaza pour un solo. D'acteur participant, le spectateur se retrouve autour du cercle comme à une première exposition de la danse. Nacera Belaza déploie, les yeux fermés, des gestes qui adviennent sous l'impulsion d'une émanation intérieure, tantôt très expressifs, tantôt tenus à l'infime, le plus souvent «sur le fil » entre deux états, entre l'inconvenue burlesque et l'obstination idiote, jusqu'aux multiples frottements au sol qui n'ont rien d'érotique ni d'animal, éprouvant seulement un contact et une répétition, au plus bas de ce qui échoit à la gravité des corps: une terre. Et une scène ancestrale tout juste délimitée par un cercle de lumières, d'où parfois une main se tend vers le haut. On pense à Wigman, Keaton, Beckett, Tanaka, tout cela à la fois en un butô très oriental, et pourtant rien de tout cela clairement, on se laisse prendre par l'insoupçonné. Un geste expressionniste trans-méditerranéen dépourvu de tout exotisme. Le spectateur se retrouve en "désapprentissage ». Il fouille dans les cellules du corps dansant l'énergie secrète, cryptée, la nature de cette radicalité, cet extrême profond animant tout son 
corps. C'est une danse hors de toute figure amenant à se poser la question de l'espace circonscrit par le geste. Car la limite au sol ne sera jamais transgressée. Mais en contrepoint du solo, une vidéo donne à voir sur toute l'horizontalité du mur du fond le corps démultiplié de la danseuse dans le Cri, une pièce fondatrice. À l'un le multiple tend cette autre forme obstinée de gestes répétés. À ce stade du cheminement dans le MuCEM, Nacera Belazaa voulu inventer une « autre scène ».

On comprend mieux alors que, descendant encore d'un niveau, par un escalier et un couloir menant à la profondeur des douves, Nacera Belaza revienne sur son parcours, tout en invitant le spectateur à se parcourir lui-même - ici à travers une vidéo issue des Sentinelles, qui marque d'une ligne en pointillés jaunes l'animation d'une route, que le spectateur finit par quitter pour entrer au bas de l'auditorium. La planche de bois trouée d'où part un faisceau de lumière éclaire Dalila Belaza du côté des gradins, assise à une place vide, tandis que les spectateurs se trouvent assis par terre du côté du plateau. Dans cette inversion spatiale on ne se trouverait plus assujetti aux ombres de la caverne de Platon. Le spectateur découvre la danseuse à sa propre place. Elle prend la pause vibrante d'un geste ouvert, au bougé à peine perceptible, un presque-rien qui contraste avec celui de sa sœur dont la retenue était indissociabled'un corps en mouvement. Ici le corps demeure en équilibre précaire, inconfortablement assis en léger déséquilibre (un autre butô plus proche d'Hijikata). Le seuil de perception est sollicité à toute fin de ne rien discerner, ne rien cerner, ne rien arrêter par le jugement, sinon de s'ouvrir à cette ouverture de mains. Saisissante est alors ce qui apparaît comme une subversion du dispositif duchampien d'Étant donnés... ${ }^{10}$, quant à la planche trouée, et au fait qu'il y aurait quelque chose à voir.

On perce alors autrement la peau et les os de cette architecture de verre et béton. On oublie la prouesse technologique du bâtiment, la force plastique de son BFU, dit béton fibré à ultra performance, qui s'affranchit de la pesanteur, soutenu par ces tangons qui sont de véritables lances vectorisant l'espace, et laissant voir l'intérieur de son grand corps où brille la mer à travers ses ajours. On oublie le ballet des corps se dessinant en contre-jour dans les limbes du parcours la journée. On se sent ancré dans un autre temps plus profond d'un imaginaire méditerranéen, par la trouée d'une vieille planche laissant voir la profondeur d'un visage. 
MuCEM, Musée des Civilisations de l'Europe et de la Méditerranée

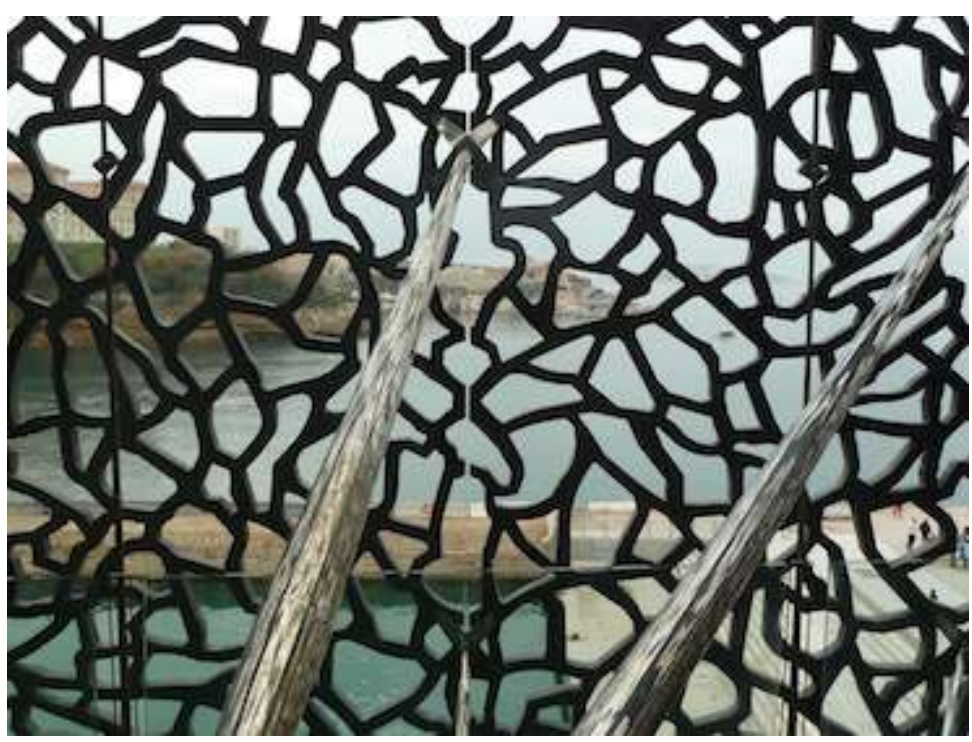

Frédérique Villemur

MuCEM, Musée des Civilisations de l'Europe et de la Méditerranée

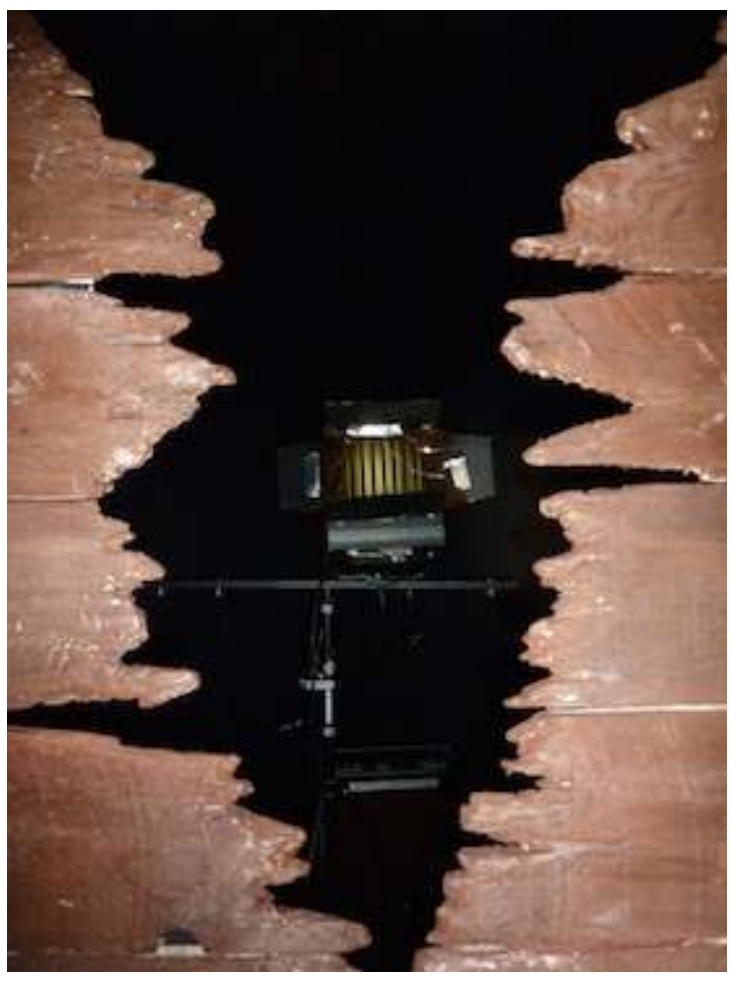

Frédérique Villemur

11 Le geste et l'expression du regard, pour théâtral qu'ils soient, l'emportent sur ce dispositif qu'on finirait par oublier si ce n'était qu'il structure l'espace qu'il éclaire, en focalisant l'attention sur Dalila Belaza. L'ouverture de ses mains aimante le regard et oriente le déplacement des spectateurs qu'elle finit par mener au-dehors de l'auditorium. La puissance de ce geste infime a réussi à faire passer une délicatesse de mouvement, attirant à lui présence et silence. 

rasante cinglent l'espace nocturne. Nacera Belaza invite le public à un autre temps de partage de la danse. Le mouvement gravitationnel de la procession trouve là son point final d'élargissement à l'espace. L'espace nu, libre de tout objet, est livré au public. Tout le monde se met à danser. Les corps se délient et se lient les uns aux autres, tournoient sur eux-mêmes, et mû par le groupe chacun finit par y déployer son propre rythme. Ce n'est pas un défouloir spectaculaire, non, tout juste donne-t-on libre cours autrement, à ce qui s'est affiné tout au long de la procession.

Pour la première fois c'est à une autre reconfiguration du sensible et de son partage que nous invitent Nacera et Dalila Belaza : la danse, en solo et collective, comme le geste performé, ont incité de manière imperceptible les spectateurs à déployer un autre espace au sein du MuCEM. La Procession, cheminement sensoriel plus que solennel, accompagné d'une bande sonore marquée par l'Oraison funèbre de Messiaen, mais aussi par Richter ou Einaudi, univers caractéristique des sœurs Belaza, se révèle être moins un parcours guidé qu'un possible déploiement d'espace reconfigurant la perception de l'architecture. Mais pas seulement, car plus profondément il se révèle être une manière d'envisager l'autre. Dans ces profondes résonnances, par-delà le tragique en partage auquel l'histoire désormais nous assigne, on pouvait ce soir-là envisager une refonte commune, par-delà les langues et les cultures. C'est en cela que le pari risqué des sœurs Belaza était gagné. Entre les objets un lien s'est trouvé, sur un fond immémoriel auquel semble ramener la danse en son geste premier, qui nous faisait être ensemble à l'espace. Rien donc ne semblait déplacé. Désacralisant l'espace muséal et réactivant une présence aux objets déplacés, $L a$ Procession revivifie un lien, celui de la survie, non pas seulement survivance où l'autrefois et l'ici ne font qu'un, ni même intensité de vie, mais bien survie dans le sens d'un partage à nouveau possible où le vivre sur ne peut être qu'un vivre entre.

\section{NOTES}

1. Rudy Ricciotti, propos recueillis par Catherine Maliszewski, Le Monde, 29 juin 2012.

2. Catherine Sentis est administratrice générale du MuCEM. À la conception du cycle « Objets déplacés » : Samar Kehdy, chargée des spectacles et arts vivants avec le concours d'Émilie Girard, conservatrice du patrimoine.

3. «Interview de Nacera Belaza pour La Procession » par Laurent Bourbousson, Ouvert-auxpublics, 15 décembre 2015.

4. Pour reprendre le titre de l'ouvrage de Mathieu Copeland (éd.), Chorégraphier l'exposition, Dijon, Les presses du réel, 2013.

5. Frédérique Villemur, Nacera Belaza, un corps entre deux rives, Actes-Sud / Éditions Barzakh, à paraître en 2017.

6. Cycle 2015-2016 : Kornél Mundruczó, Nacera Belaza, Angelica Liddell, Faustin Linyekula.

7. Éditorial du programme « Objets déplacés », p. 4.

8. Avec toute l'ambiguïté que porte ici cette expression : Idem, Fabienne Arvers, p. 5. 
9. On reconnaît une meule en pierre " à la matière vivante " note Nacera Belaza, une maie de pressoir dont le trou lui évoque "un point de fuite ", ainsi que le détail d'un tableau de la deuxième moitié du XIXe siècle, Femmes en prière, de Victor Lainé, choisi par Dalila Belaza. Mais ces informations ne sont pas livrées au public.

10. Marcel Duchamp, Étant donnés : $1^{\circ}$ la chute d'eau $2^{\circ}$ le gaz d'éclairage, 1946-1966, installation, Philadelphia Museum of Art. 